\title{
DISEGUAGLIANZE ECONOMICHE, SQUILIBRI AMBIENTALI E FLUSSI MIGRATORI
}

\author{
Simona Pisanelli \\ Dipartamento di Storia, Società e Studi sull'Uomo \\ Università del Salento
}

\begin{abstract}
L'obiettivo di questo paper è quello di rilevare l'inadeguatezza dell'economics rispetto alla spiegazione della relazione esistente tra i problemi relativi allo sviluppo, agli squilibri ambientali e ai flussi migratori. Mentre questi tre ordini di problemi, presi singolarmente, sono spesso al centro di discussioni pubbliche e politiche, sul piano analitico manca ancora un soddisfacente approccio in grado di spiegarne connessioni causali e implicazioni sociali. Ciò appare sorprendente, specie se si considera che già gli Illuministi avevano avviato una ricca riflessione su questi temi. Si cercherà di dimostrare che l'impoverimento analitico è sopraggiunto nel XIX secolo, con l'adozione della categoria dell'homo oeconomicus e che tale impoverimento, ad oggi, impedisce di spiegare fenomeni come le "migrazioni ambientali" non ancora pienamente e univocamente riconosciute in letteratura.
\end{abstract}

Parole chiave: sviluppo, giustizia sociale, cambiamenti climatici, migrazioni, Illuminismo

\section{Economic inequalities, environmental imbalances and migration flows}

\begin{abstract}
The aim of this paper is to point out how inappropriate economics is for explaining the relationship between development problems, environmental imbalances and migration flows. While these problems, taken individually, are often the focus of public and political discussions, there is still no satisfactory analytical approach able to deal with their causal connections and social implications. This seems surprising, especially if one considers that the Enlightenment thinkers were already engaged in a rich reflection on these issues. In this paper we will try to demonstrate that a kind of analytical impoverishment occurred in the nineteenth century, with the adoption of the homo oeconomicus category and that such an impoverishment, nowadays, prevents us from explaining phenomena such as "environmental migrations", which are not yet fully and unambiguously recognized in economic literature.
\end{abstract}

Keywords: development, social justice, climate change, migrations, Enlightenment 


\section{Introduzione}

Quando si affrontano con continuità problemi come quelli dell'ambiente, dell'emigrazione e dei flussi migratori dovuti agli squilibri ambientali si incappa in risultati che paiono spesso o non ragionevolmente dimostrati o contraddittori. Si ha l'impressione che più che spiegare l'insieme dei fenomeni sul tappeto, indagandone la genesi e le relazioni causali significative, prevalga un atteggiamento descrittivo, magari connotato ideologicamente, in cui di volta in volta vengono introdotti fattori esplicativi ad hoc. Riflessioni più approfondite evidenziano la inevitabilità di questi esiti analitici, che paiono collegati ad approcci epistemologici inadeguati in quanto non dimensionati alla natura degli oggetti indagati. La cosa pare particolarmente significativa se si considera il modo in cui l'economics pretende di affrontare questo tipo di problemi senza alterare il suo approccio epistemologico. Essa, infatti, continua a porre al centro del discorso l'homo oeconomicus e il peculiare rapporto tra individui ed economia, individui e societâ che esso rende possibili. II suo intento non è quello di confrontarsi con i temi e i rischi che una certa organizzazione dell'economia e della società pongono in una determinata fase della loro evoluzione ma muoversi lungo l'asse bisogniuniverso di beni a disposizione, onde massimizzare i vantaggi individuali.

Già Mary Douglas si chiedeva come mai questo «perfetto estraneo» sia arrivato a dominare la nostra riflessione su noi stessi e sui nostri rapporti con il mondo. Il fatto è, rilevava l'antropologa, che l'homo oeconomicus, per le caratteristiche che lo contraddistinguono e per il tipo di razionalità che ne definisce i comportamenti, non può aiutarci a ricostruire i sistemi di relazioni che caratterizzano gli universi storici in cui l'vomo vive e agisce. Egli, infatti, manca di caratteri adeguati per tale scopo: preoccupato di massimizzare i suoi livelli di utilità non è dotato di «attributi sociali» (Douglas 1995, p. 5), evidenzia una radicale insensibilità verso i problemi di «equità» (Douglas 1995, p. 6) e manca di quella immaginazione e creatività che caratterizzano gli esseri sociali inseriti nel flusso del divenire storico e che può determinare fenomeni innovativi che non possono ridursi agli aspetti cumulativi tipici dell'homo oeconomicus.

Attraverso questo atteggiamento epistemologico e tramite la categoria dell'homo oeconomicus vengono introdotti i seguenti presupposti: gli individui sono caratterizzati esclusivamente dalla propensione a soddisfare i propri bisogni; essi, nello spazio del mercato, si muovono come agenti che hanno gli stessi attributi e lo stesso potere (le diversità sono riconducibili solo a differenze di reddito dei singoli) e, infine, non mostrano alcun interesse per quanto riguarda la società e l'ambiente. Ovviamente, come diceva J. Stuart Mill, introducendo la centralità della categoria dell'homo oeconomicus nell'analisi economica, ciò non significa che gli individui "reali" siano fatti in questo modo. L'economista non è tanto folle da trarre queste conclusioni; il problema è che la scienza deve lavorare con un approccio 
esclusivamente deduttivo e la categoria dell'homo oeconomicus si dimostra efficace nella spiegazione dell'agire economico. Ciò che Mill sottovaluta è il fatto che questa categoria, adottata per comodità analitiche (Keynes 1996, p. 31ss), diviene - come rilevava Douglas - un punto di riferimento per tutte le scienze sociali, portando a forme di riduzionismo che impoveriscono la stessa interpretazione dei fatti economici. In tal modo, il suo uso porta a spiegazioni che finiscono per occultare aspetti della realtà che sono essenziali per comprendere la dinamica sociale. In questo contributo si vuole dimostrare quanto segue:

1. questo impoverimento analitico non è il risultato inevitabile di una scelta epistemologica neutra, ma l'effetto di un modo specifico di osservare la realtà;

2. la riflessione illuministica su questi temi è stata molto più ricca e ha tentato di costruire un modello di scienza in grado di valutare la complessità dell'agire e la complessità delle articolazioni economiche e sociali che la scienza deve analizzare;

3. che un approccio fondato sull'homo oeconomicus e sulle sue propensioni alla massimizzazione dei vantaggi individuali, anche in contrasto con le esigenze sociali, da un lato non consente di cogliere il tema della giustizia e dell'equità sociale; dall'altro, lascia sullo sfondo la relazione tra ambiente naturale e evoluzione sociale;

4. che ciò ha effetti significativi per quanto concerne lo studio dei fenomeni migratori che sono determinati dall'arretratezza economica che coinvolge segmenti significativi della popolazione mondiale, senza sottovalutare l'impatto negativo che gli squilibri ambientali hanno sulle condizioni di vita di intere aree della terra.

\section{Agire individuale, equità e sviluppo sociale: le riflessioni illuministiche}

Ovviamente, in questa sede non si vogliono considerare i risultati delle riflessioni dei pensatori dell'llluminismo come un punto di riferimento per individuare la soluzione dei problemi che caratterizzano il mondo contemporaneo. II tema che si vuole porre qui è se, ancora oggi e al di là dei limiti che hanno caratterizzato questi pensatori (Diderot, Smith, Kant, Condorcet, ecc.), il loro approccio epistemologico possa essere di una qualche utilità per l'analisi del mondo contemporaneo e dei rischi che esso presenta.

Ora, contrariamente a quello che generalmente si crede, i pensatori illuministici non hanno mai pensato che la crescita della ricchezza materiale rappresentasse il fine ultimo dell'agire umano. Ovviamente, essi hanno enfatizzato il ruolo positivo della crescita economica, considerandola come una precondizione dell'emancipazione degli vomini dalla povertà e dai bisogni materiali. Tuttavia, al centro dei loro interessi troviamo il tema dello sviluppo umano, quello, declinato in modi diversi, dell'uguaglianza tra gli uomini e quello di un progresso sociale in grado di coinvolgere tutti i paesi. 
II tema della crescita non era affrontato in modo riduttivo, immaginando individui che aumentassero progressivamente la quantità di beni a loro disposizione. Esso veniva analizzato a partire dall'ipotesi che la ricerca della "felicità", che caratterizza tutti gli individui, dovesse porsi nel contesto di un rapporto virtuoso con la società: "felicità privata" e "felicità pubblica" erano, nella loro prospettiva, inscindibili. Se la prima avesse avuto il sopravvento, la ricerca ossessiva della "felicità privata" avrebbe prodotto la riduzione del benessere pubblico, che della prima era una precondizione essenziale; d'altra parte, se la "felicità pubblica" avesse avuto la priorità, si sarebbe corso il rischio di impoverire i caratteri della moderna individualità, con effetti negativi - a lungo andare - anche per il progresso sociale (sono temi che sono stati ripresi, tra gli altri, da teorici come Galbraith 1958, Galbraith 1993, Sen 1988, spec. pp. 75 - 109, Sen 2000). È indicativo di questo atteggiamento, quanto scrive Diderot:

«... I'homme qui n'écoute que sa volonté particulière est l'ennemi du genre humain: .. la volonté générale est dans chaque individu un acte pur de l'entendement qui raisonne dans le silence des passions sur ce que l'homme peut exiger de son semblable, et sur ce que son semblable est en droit d'exiger de lui ; ... cette considération de la volonté générale de l'espèce et du désir commun est la règle de la conduite relative d'un particulier à un particulier dans la même société, d'un particulier envers la société dont il est membre, et de la société dont il est membre envers les autres sociétés; ... que la soumission à la volonté générale est le lien de toutes les sociétés, sans en excepter celles qui sont formées par le crime ...) (Diderot 1782, p. 371).

Così quando Adam Smith individua i caratteri della moderna "società commerciale" e la caratterizza come una società progressiva (al di là delle diseguaglianze in essa esistenti), lo fa in base alla considerazione che essa avrebbe contribuito a creare una situazione in cui non solo gli abitanti delle nazioni avanzate, ma anche quella degli altri paesi sarebbero pervenuti a uno stato di eguaglianza, attraverso lo scambio reciproco di conoscenze e progressi di ogni tipo, favoriti anche dal commercio internazionale. Ne L'indagine sulla natura e le cause della ricchezza delle nazioni (1776), l'autore poneva in evidenza i diversi livelli di sviluppo raggiunti dai Paesi dell'occidente europeo e quello di altri continenti, ma non si rassegnava ad accettare tale stato del mondo come immodificabile. Secondo la sua convinzione, la generalizzazione della nuova organizzazione produttiva avrebbe prodotto non solo l'incremento della ricchezza materiale, ma anche lo sviluppo crescente della consapevolezza degli attori sociali (se non altro perché essa implicava necessariamente lo sviluppo della scienza e delle sue applicazioni tecnologiche all'economia e alla società), determinando positive trasformazioni strutturali.

Non è certo casuale se autori come Kant (tanto per citare uno dei più grandi teorici dell'llluminismo) immaginavano un sistema di possibili eguaglianze che dai cittadini all'interno di uno stato potesse essere trasferito ai rapporti tra gli stati. Nel 1795 - nel suo scritto Per la pace perpetua - ipotizzava che il clima di benessere e razionalità che si diffondeva nell'epoca dei Lumi avrebbe favorito la propensione degli Stati a rinunciare al perseguimento esclusivo dei propri interessi e alle forme di 
competizione internazionale sino ad allora sperimentate per aprirsi a esperienze che ponessero lo sviluppo equo e pacifico al centro delle politiche nazionali. Anche in questo caso, il progetto di cittadinanza globale veniva considerato possibile, a condizione che si superasse la netta divisione tra ricchi e poveri in virtù della generalizzazione degli effetti del progresso economico e sociale. II tema dell'equità e della giustizia sociale rappresentava certamente il nucleo fondamentale del discorso kantiano.

È un atteggiamento condiviso anche dall'ultimo dei philosophes, Condorcet, il quale si distingue dagli altri per una spiccata propensione a considerare - quasi nei termini di un programma ben determinato e realizzabile ${ }^{1}$ - la società di eguali, «attribuendole un carattere di prevedibilità, certezza e scientificità e trasformandola in un processo immanente di composizione delle molteplicità delle autonomie individuali e dell'eguaglianza universaleı (Durante 2009, p. 7). La realizzazione dell'eguaglianza tra gli uomini viene posta come presupposto del discorso teorico e come fine del'agire umano nell'ipotesi che si possa perseguire concretamente il perfezionamento indefinito dell'vomo.

L'atteggiamento riformatore di Condorcet si evidenzia nelle sue molteplici proposte di riforma (alla base delle quali pone l'irrinunciabilità dei diritti fondamentali dell'vomo, in continua e permanente espansione, e la legittimità del potere fondata su tali diritti) imperniate sull'esigenza di abbattere i diversi tipi di diseguaglianza esistenti nella società del suo tempo:

il riconoscimento dell'eguaglianza giuridica e politica tra i due sessi abbatterà la diseguaglianza tra vomo e donna;

l'affrancamento graduale di schiavi e il superamento definitivo del razzismo eliminerà la diseguaglianza tra bianchi e neri;

il modello repubblicano e un sistema democratico basato sul suffragio universale limeranno la diseguaglianza tra sovrano e suddito;

- $\quad$ il processo egualitario del possesso di proprietà avvicinerà ricchi e poveri;

- $\quad$ infine, un programma di istruzione pubblica esteso a tutti i cittadini e tra le varie nazioni, cancellerà la diseguaglianza tra illuminati e incolti.

Proprio l'istruzione pubblica, che dovrebbe fornire ai cittadini gli strumenti di critica per giudicare la propria attività, è indicata da Condorcet come lo strumento più idoneo per sradicare le diseguaglianze. Un cittadino che sia correttamente istruito potrà esercitare liberamente la ragione e appellarvisi costantemente, nonché esprimere giudizi critici sull'operato di quanti lo governano.

Il programma universale di istruzione serve a strappare la popoulance (moltitudine di vomini incolti) alla pericolosa influenza delle «forze reazionarie, dai preti agli vomini di legge, ai nuovi borghesi in affari» (Durante 2009, p. 24). I progressi nelle scienze assicurano il progresso nell'istruzione e viceversa, producendo un circolo

1 Voltaire, per esempio, nell'articolo Egalité del suo Dictionnaire philosophique (1764) scriveva che «l'eguaglianza è [...] al tempo stesso la cosa più naturale in linea di diritto, e la più chimerica in fatto» (Voltaire 1965 , p. 254). 
virtuoso che contribuisce al perfezionamento della specie umana. II continuo avanzamento degli uomini si riversa nelle arti e nelle tecniche produttive: strumenti, macchine e mestieri accrescono l'abilità dell'vomo nell'ottenere prodotti migliori, impiegando minore quantità di lavoro in minor tempo, senza trascurare miglioramenti in termini di abitudini, clima e salubrità. Appezzamenti di terreno sempre più piccoli riescono a soddisfare i bisogni di più persone, mentre il prodotto dell'industria richiede minore distruzione di materie prime o diviene di uso più durevole.

D'altro canto, lo sviluppo di tali facoltà può comportare anche rischi. Al pari di Malthus, ma in termini meno catastrofici, Condorcet rivela una certa preoccupazione per l'eccessiva crescita della popolazione. In un passo della sua ultima opera, I progressi dello spirito umano (1795), si legge:

uin questi progressi del lavoro e del benessere, da cui risulta un rapporto più vantaggioso tra le facoltà dell'vomo e i suoi bisogni, ogni generazione, sia per questi progressi, sia per la conservazione dei prodotti di un'attività anteriore, è chiamata a godere di vantaggi più ampi, e quindi, in conseguenza della costituzione fisica della specie umana, ad un aumento del numero degli individui; allora, non deve forse giungere un momento in cui queste leggi, ugualmente necessarie, verrebbero a contrapporsi; in cui, superando l'aumento degli vomini quello dei loro mezzi, ne risulterebbe necessariamente, se non una diminuzione continua del benessere e della popolazione o una marcia veramente a ritroso, almeno una sorta di oscillazione tra il bene e il male?») (Condorcet 1995, p. 199).

Ebbene, Condorcet conserva la speranza anche di fronte a un simile scenario: se mai dovesse giungere un'epoca simile, egli crede che - nel frattempo - la ragione dell'vomo sarà progredita di pari passo con le scienze e con le arti, la morale si sarà liberata da pregiudizi e da superstizioni, così che gli individui si sentiranno obbligati a garantire la felicità anche alle generazioni future. Per assolvere questo dovere essi potrebbero porre un limite alla quantità degli alimenti e, di conseguenza, al tetto massimo della popolazione. In sostanza, Condorcet preventiva una riduzione volontaria del tasso di natalità, prevedendo un'epoca in cui le famiglie di piccole dimensioni saranno il frutto di una scelta consapevole, dettata dal progresso della ragione; quest'ultimo, a sua volta, sarà il risultato dell'espansione dell'istruzione, specie di quella femminile (Condorcet 1995, p. 203).

In questa sede non è utile soffermarsi sui limiti della visione illuministica, né sul fatto che - dati i tempi in cui essa è stata formulata - il riferimento all'ambiente è marginale. Tuttavia, sul piano epistemologico emerge un elemento realmente significativo:

1. nell'evoluzione della società, l'economia ha assunto un ruolo preponderante, mostrando la capacità di determinare incrementi della ricchezza inimmaginabili nelle precedenti organizzazioni sociali;

2. la crescita economica appare (anche per esigenze endogene come il perfezionamento dell'organizzazione produttiva e l'uso sempre maggiore della 
scienza) come una condizione di un processo di emancipazione dell'vomo sempre più ampia e profonda;

3. tuttavia, resta il fatto che la crescita della ricchezza materiale va giudicata non in sé, ma nel contesto di una visione che pone equità e giustizia sociale al centro del discorso.

Ora, al di là dei limiti della riflessione illuministica, la centralità di questo elemento appare essenziale anche per ridefinire i termini del discorso sul rapporto individuisocietà e società-ambiente naturale. Come rilevato da Mahbub ul Haq2:

«In economic science, nothing is ever new, and nothing permanent; Ideas emerge, flourish, wither and die, to be born again a few decades later. Such is the case for ideas about human development. The founders of economic thought never forgot that the real objective that the real objective of development was to benefit people, creating wealth was only a means. That is why, in classical economic literature, the preoccupations is with all of society, not just with the economy. Fascination with industrial chimneys and technology did not replace early economists' concern with real peoples (Haq 2003, p. 11).

Per questa ragione tornare ai classici non ha un intento puramente archeologico, ma suppone il bisogno di tornare a un paradigma che rimetta al centro dell'interesse degli analisti ureal people» e «social evolution».

\section{Una nuova definizione di eguaglianza: la giustizia ecologica}

A livello planetario, il principio dell'eguaglianza attraverso lo sviluppo si è rivelato, purtroppo, una promessa mancata. Se è vero che, nei decenni immediatamente successivi alla seconda guerra mondiale, gli Stati ricchi hanno assicurato il benessere dei propri cittadini attraverso posti di lavoro e redistribuzione del surplus (anche per effetto di una sorta di patto tacito tra Stato e grandi imprese che aveva caratterizzato il periodo di produzione fordista), è vero che nello stesso tempo si è ampliato il «divario ... tra la costituzione di un immaginario collettivo da parte della società dell'informazione e la realtà territoriale della spartizione tra ricchezza e povertà. [...] questo divorzio non avviene solo tra paesi ricchi e paesi poveri, ma caratterizza anche gli stessi paesi ricchi in cui l'opposizione centro/periferia si sostituisce all'antico schema della lotta di classe all'interno dell'azienda stessa») (Cohen 2004, p. 66). Fatta eccezione per alcuni casi particolari, il quadro globale restituisce ancora l'immagine di un mondo attraversato da profonde diseguaglianze.

Quando si riflette sugli squilibri mondiali, si corre il rischio che - definendo le differenze di ricchezza tra Paesi come elemento di ineguaglianza e di ingiustizia - si

\footnotetext{
${ }^{2}$ Mahbub ul Haq è l'economista che, insieme con un gruppo di lavoro, composto da diversi studiosi, tra i quali Amartya Sen, ha elaborato lo Human Development Index (HDI) che è alla base delle analisi condotte annualmente dall'UNDP (Programma per lo sviluppo delle Nazioni Unite) sulla qualità della vita e sul benessere dei cittadini.
} 
finisca per ritenere che tutti i Paesi debbano far parte di uno stesso sistema economico. L'idea veicolata è che si può ottenere eguaglianza sociale grazie allo sviluppo economico accelerato e permanente, livellando verso I'alto, mai verso il basso, le posizioni dei singoli Paesi (Douglas Lummis in Sachs 1998, pp. 410-1). Così facendo, si persegue l'eguaglianza nell'accezione di similitudine o omogeneità (homoios), anziché in termini di giustizia o trattamento equo (isos), costringendo tutti i popoli del mondo in un unico sistema economico e culturale.

In ogni caso, «da quando i limiti della natura sono diventati visibili, le stesse coordinate della giustizia sono cambiaten (Sachs 2002, p. 27). Per lungo tempo, la questione della giustizia sociale è intesa come risolvibile attraverso gli strumenti del progresso e della crescita economica. Decisamente improntata all'ottimismo, la tradizione di pensiero che risale ai primi dell'Ottocento, non prevede limiti all'aumento della ricchezza. Finché si accetta per buona l'idea di uno sviluppo senza freni in termini esclusivamente materiali, "allora è possibile concepire la giustizia, sia sul piano nazionale che internazionale, come partecipazione sempre maggiore di una fascia sempre più ampia di popolazione a un surplus anch'esso sempre più grande»33 (Sachs 2002, p. 181). Autolimitazione della crescita e redistribuzione della ricchezza sono, entrambe, registrate come ostacolo allo sviluppo.

Con la crisi ecologica, con l'emergere dei limiti biofisici alla crescita economica con i quali si "scopre" che le fonti non monetarie di ricchezza non sono infinite e i beni naturali non sono inesauribili - diventa inevitabile ridefinire le condizioni per la creazione della ricchezza in futuro. Il progresso materiale non è illimitato, ma deve adeguarsi ai vincoli imposti dall'ambiente. "La prospettiva di una maggiore equità non può più intendersi nel senso di una crescita continua. [...] Essa può essere democratizzata solo a costo della distruzione della biosfera» (Sachs 2002, p. 183).

Il punto è che le pressioni da parte dei Paesi che finora hanno subito le maggiori deprivazioni non accennano, giustamente, a diminuire. La divisione del mondo in Paesi industrializzati e Paesi in via di sviluppo che ha tenuto banco nella seconda metà del secolo scorso, rappresenta un modello obsoleto. I Paesi emergenti - o, meglio, ex emergenti quando si parla di casi come Cina e India - reclamano maggiore giustizia sociale, non solo in termini di maggiore benessere, ma anche come confronto da pari a pari con le vecchie potenze.

Questo desiderio di imitazione dello stile di vita occidentale è diffuso, prima ancora che a livello nazionale, tra le singole persone che riconoscono nelle merci consumate in Europa occidentale o negli Stati Uniti il simbolo del benessere, così come identificano nell'aumento di città, acciaierie, autostrade, agricoltura intensiva tutti elementi di successo. Secondo alcuni autorit, la decolonizzazione dell'immaginario è più urgente e utile di qualsiasi decolonizzazione politica o economica, perché l'immaginario cui si ispirano i Paesi emergenti non è più

\footnotetext{
${ }^{3}$ È evidente che alla base di questa modalità di intendere la giustizia c'è il famoso slogan utilitarista di Jeremy Bentham, "la massima felicità per il massimo numero", contenuto nella sua Introduction to the Principles of Morals (1780).

${ }^{4}$ Si veda, a tale proposito, Latouche 2000; Latouche 2005; Sachs-Morosini 2011.
} 
realizzabile nel presente. La crescita straordinaria che ha investito la civiltà euroatlantica è legata a circostanze storiche uniche, non più ripetibili, perché caratterizzata dall'abbondanza di combustibili fossili (oggi pressoché in esaurimento) e dalla possibilità di sfruttamento delle colonie.

A oggi, ciò che resta è un equilibrio ecologico globale fortemente compromesso dall'uso indiscriminato delle risorse non rinnovabili, impiegate nei processi industriali di produzione, e dalla difficoltà di contenere le emissioni dannose per la qualità dell'aria e dell'acqua e dallo smaltimento dei rifiuti derivanti tanto dai cicli produttivi quanto dal consumismo.

I più ottimisti hanno accettato la tesi della Environmental Kuznets Curve, in base alla quale una relazione a $U$ legherebbe lo sviluppo economico e lo sviluppo ambientale: con l'aumento del reddito pro capite l'impatto ambientale aumenta solo in una prima fase, per poi tornare a diminuire grazie alla maggiore sensibilità ecologica, a un'accresciuta domanda di qualità ambientale, a maggiori risorse finanziarie destinate alle politiche ambientali e soprattutto, si sostiene, al progresso tecnico. In realtà, quest'ultimo non è necessariamente positivo. Nasconde, anzi, un lato estremamente insidioso, poiché i miglioramenti d'efficienza non portano necessariamente e automaticamente alla riduzione generale dei consumi.

Tuttavia, l'attenzione per i problemi legati allo stile di vita consumistico (prettamente occidentale) è stata, a lungo, relegata nell'ambito dell'ecologia. Si pensava a essi come una minaccia "solo" per il clima. Quando, nel 2006, il Rapporto Stern ${ }^{5}$ ha dichiarato che il cambiamento climatico - se non fermato in tempo - avrebbe procurato uuna perdita complessiva del PIL del $20 \%$ pari all'impatto negativo delle due ultime guerre mondiali messe assiemen (Niada 2006), la preoccupazione è divenuta d'interesse generale, senza però tradursi in interventi decisivi e risolutivi rispetto al rischio di oltrepassare la capacità di resistenza dei sistemi naturali.

Infatti, mentre la letteratura sul tema delle diseguaglianze cresce in maniera straordinaria e, in modo altrettanto rilevante, cresce la letteratura sul tema degli squilibri ambientali e sull'impatto che essi avranno sulla dinamica delle nostre società, l'economics continua ad analizzare il sistema economico con un approccio che non solo appare arretrato rispetto ai problemi che é chiamata a risolvere, ma evidenzia una sostanziale tendenza elusiva (rinviando la necessità urgente di politiche nazionali e internazionali che comincino a sintonizzarsi con il tema degli squilibri ambientali).

È sorprendente che, ha rilevato Sen, malgrado l'impressionante mole di dati e di analisi sugli squilibri ambientali «sono ancora poco conosciute la misura esatta del rischio ambientale e le conseguenze che potrebbero venircene - a meno che non lo contrastiamo» (Sen 2002, p. 97). Inoltre, non sappiamo nemmeno "quando dovremmo attendere gli effetti disastrosi che ci minacciano e come potremmo agire» (ibidem).

\footnotetext{
${ }^{5}$ Rapporto commissionato dal governo inglese a sir Nicholas Stern, ex capo economista della Banca Mondiale.
} 
Tuttavia, ciò che sembra ancora più paradossale è il fatto che sfugga la rilevanza epistemologica, oltre che pratica, della "questione ambientale". In effetti, un'appropriata tematizzazione del rapporto tra dinamica economico-sociale e contesto ambientale consentirebbe di avere una visione unitaria dell'insieme dei problemi che la scienza economica e le altre scienze sociali devono affrontare, se vogliono definire una teoria dello sviluppo nel quadro di un rinnovato rapporto tra vomo e ambiente naturale. Per la prima volta, infatti, la percezione della dimensione sistemica e planetaria della questione ambientale costringe a considerare lo sviluppo non in termini di meccanica estensione di un modello di successo (quello occidentale), bensì in termini di un radicale mutamento del punto di vista, in grado di valutare i paesi sviluppati e i paesi arretrati come due aspetti di un medesimo problema. Si tratterebbe di un vero e proprio cambio di paradigma, che porrebbe le variabili economiche non solo in relazione con quelle sociali, ma soprattutto in relazione al rischio ambientale. Esse, cioè, pur avendo come punto di riferimento situazioni nazionali o regionali, dovrebbero essere immediatamente valutate nel quadro di un sistema di compatibilità definito su scala planetaria (Bologna 2005, p. 94).

L'analisi dei problemi ambientali (siano essi relativi all'inquinamento crescente, all'esaurimento delle risorse naturali e allo spreco di fattori determinanti per la vita delle future generazioni) presuppone una visione di lungo periodo (contraria alla logica massimizzante e di breve periodo dell'homo oeconomicus) e un'analisi sistemica dei rischi degli squilibri ambientali. Questi due aspetti non possono entrare nella prospettiva dell'homo oeconomicus o nei criteri di razionalità che egli adotta nel suo ossessivo, meccanico e ripetitivo rapporto con l'universo di beni necessari per il soddisfacimento dei suoi bisogni. Tuttavia, se l'economics continua a considerare al centro del suo paradigma l'homo oeconomicus e il suo rapporto con il mercato, è difficile che si apra una reale riflessione sull'ambiente naturale, sugli squilibri indotti dai processi economici nell'attuale configurazione. All'interno del paradigma adottato dall'economics si evidenzia l'incapacità di rinunciare alle forme di razionalità che caratterizzano il suo modello di individualismo, ma anche la convinzione che un sistema economico e sociale, considerato il migliore possibile con lo sguardo rivolto al passato - possa cambiare.

Ovviamente, non si può evitare di dedicare analisi relative alla "questione ambientale", ma nemmeno affrontarlo esclusivamente - anche se con tecniche analitiche molto sofisticate - nel quadro di un approccio epistemologico che continua ad avere al centro del discorso l'homo oeconomicus e le sue propensioni massimizzanti (che possono essere solo parzialmente modificate da scelte relative alla salvaguardia ambientale). Tuttavia, sembra diventare sempre più evidente che il rapporto vomo-natura e il tema del cambiamento climatico non possono essere affrontati entro le coordinate dell'approccio epistemologico mainstream e con la strumentazione analitica che esso mette a disposizione degli altri scienziati sociali (oltre che dell'economista di professione). Normalmente, rileva Dimitri Zenghelis, questi temi vengono affrontati «sia dagli economisti che dai responsabili delle politiche, come un "problema ambientale", valutabile mediante gli strumenti tradizionali di analisi e politica economica») coeteris paribus (Zenghelis 2017, p. 277). 
Si conferma, cioè, la tendenza a ignorarne aspetti significativi: in primo luogo, il fatto che oggi non è possibile stimare con precisione gli effetti che il global warming può avere non su aspetti singoli o specifici della vita economica, ma sulle possibilità di esistenza della specie umana; in secondo luogo, che per frenare o per lo meno tenere sotto controllo gli effetti delle crisi ambientali, sia necessario mettersi in una prospettiva di cambiamenti radicali assai differenti rispetto a quelli sino a oggi adottati. In particolare, per ritornare alle considerazioni di Zenghelis:

1. «affrontare i cambiamenti climatici richiede una trasformazione molto profonda dei sistemi economici, e questo impone a sua volta una prospettiva più ampia ed evolutiva»' (Zenghelis 2017, p. 277);

2. bisogna essere in grado di valutare gli effetti cumulativi degli squilibri, comprendendo ula natura path-dependent (influenzata dall'andamento pregresso) del progresso tecnologicon (Zenghelis 2017, p. 277). D'altra parte, "the climate system" è complesso e non-lineare. Le sue interazioni sono tăli che studiare effetti particolari, isolandoli dal contesto sistemico, può produrre non solo errori analitici rilevanti, ma anche errori di prospettiva a sostegno di atteggiamenti e politiche tranquillizzanti dalle conseguenze disastrose;

3. bisogna uripensare l'uso e la progettazione dei modelli economici e mettere in campo una gamma di strumenti economici molto più ampia di quella tradizionale "cassetta degli attrezzi"), cui l'economista ricorre ordinariamente sulla base del proprio paradigma scientifico (Zenghelis 2017, p. 277).

L'alto livello di diseguaglianze tra diverse aree del mondo è reso evidente, specie negli ultimi decenni, non solo dall'intensità dei flussi migratori, ma anche dalla crescente percentuale degli stessi attribuibili agli effetti dei cambiamenti climatici: il riferimento è alle cosiddette migrazioni ambientali.

\section{Squilibri ambientali e nuovi flussi migratori}

Era il 1992, quanto il Programma delle Nazioni Unite per l'Ambiente (UNEP) stimava 50 milioni di possibili rifugiati ambientali, dovuti a tre fattori principali:

«firstly the effects of warming in some areas will gradually reduce agricultural productivity and degrade ecosystem services such as clean water and fertile soil. Secondly, the increase in extreme weather events - especially heavy rainfall and resulting flash or river floods in tropical regions - will affect increasing numbers of people, resulting in mass displacement. Thirdly, sea-level rise will permanently destroy extensive low-lying coastal areas - resulting in the permanent relocation of millions of people») (Zetter 2015, p. 4).

In effetti, l'aumento generalizzato della temperatura ha provocato lo scioglimento dei ghiacciai, I'aumento della temperatura dell'acqua e, necessariamente, 
I'innalzamento del livello dei mari. Ogni anno, gli oceani si innalzano di 3,4 millimetri, tanto che - dal 1993 a oggi - la crescita complessiva ammonta a 8,48 centimetri. I primi a decidere di migrare, dunque, sono certamente i residenti sulle coste, seguiti da coloro che vivono nell'entroterra.

Sull'argomento, I'UNEP è tornato più volte. Basti pensare allo studio From Conflict to Peacebuilding. The Role of Natural Resources and the Environment che, nel 2009, ha ispirato la relazione presentata da Per Stig Møller, ministro degli esteri danese, ai suoi omologhi di altre nazioni e ai ministri dell'ambiente. Nello stesso periodo, si svolgevano gli incontri di preparazione alla Conferenza Internazionale di Copenaghen sui cambiamenti climatici. Tra le altre cose, il discorso di Møller sottolineava che ule migrazioni saranno una sfida sempre più seria, collegata al problema dei cambiamenti climatici e a quello della sicurezza. Sarà probabilmente quello il principale impatto sociale dei cambiamenti climatici e rappresenterà forse la sfida più importante che questi determineranno in termini di sicurezza in Europa» (CeSPI 2010, p. 4). È compito, dunque, del vecchio continente prendere coscienza dell'importanza crescente che i movimenti migratori causati dai cambiamenti climatici va assumendo (anche perché il rapporto è biunivoco e le immigrazioni possono influire sull'alterazione dell'ambiente che le riceve). Tali movimenti migratori, ovviamente, non derivano solo da mutamenti strettamente ambientali che colpiscono i paesi in cui hanno origine, ma sono provocati da una serie di altre cause, sempre connesse ai cambiamenti climatici: tra queste, si considerino conflitti per le risorse, danni e rischi per città costiere e infrastrutture, dispute sulle frontiere e perdite di territorio, ecc. La gravità della situazione è testimoniata da un dato molto significativo fornito nel report Climate Change and International Security: "the UN estimates that all but one of its emergency appeals for humanitarian aid in 2007 were climate related) (High Representative and the European Commission to the European Council 2008, p. 1; corsivo aggiunto).

Un decennio dopo, questo dato sembra confermato dalle poco incoraggianti stime dell'Alto commissariato delle Nazioni Unite per i rifugiati (UNHCR): entro il 2050 i rifugiati ambientali potrebbero persino essere compresi tra 200 e 250 milioni di persone fuggite dalle zone vulnerabili a causa dell'innalzamento del fivello del mare, di tempeste o inondazioni o di terreni agricoli divenuti troppo aridi per essere coltivati. Con riferimento allo stesso anno, l'Organizzazione internazionale per le migrazioni (OIM) prevede quota un miliardo di migranti ambientali.

Nonostante l'abbondanza di previsioni dei fenomeni migratori di questo tipo (non sempre coerenti tra loro) da un punto di vista metodologico della ricerca, restano evidenti le difficoltà legate alla capacità di quantificare con esattezza a quanto ammonta la percentuale di migrazioni provocate specificatamente da motivi ambientali. L'opinione del CeSPI a tal proposito è che «le stime sulle migrazioni indotte dai cambiamenti climatici sono molto incerte» e che «la crisi ambientale è probabilmente destinata a sommarsi alle principali determinanti dei processi migratori, a cominciare dalla ricerca di lavoro, piuttosto che causare nuovi flussiin (CeSPI 2010, p. 20). Le ragioni che ostacolano l'isolamento dei mutamenti climatici e ambientali come causa principale delle migrazioni (già avvenute in passato e attualmente in corso) sono sostanzialmente due: 
a) per un verso, è difficile dimostrare il nesso causale diretto tra cambiamento climatico e necessità migratoria e sembra quasi impossibile isolare questa causa specifica dalle altre concause (economiche, sociali, politiche, demografiche). Si consideri poi che il legame tra disastri ambientali e movimenti migratori non è così diretto, poiché upeople with low to medium asset levels often become trapped, in their homes during disasters or find that their vulnerability increases where they have been displaced, following a disastern (Zetter 2015, p. 5);

b) per un altro verso, i fattori ambientali come causa delle migrazioni sono ignorati nella letteratura più propriamente scientifica. Non è un caso che, a lungo, essa non sia riuscita a convergere su una definizione univoca di "migrante ambientale"6. Si possono genericamente includere in questa categoria coloro che sono costretti a lasciare il loro tradizionale habitat, temporaneamente o permanentemente, a causa di marcate disgregazioni ambientali (naturali e/o di origine antropica) che hanno messo in pericolo la loro esistenza e/o seriamente colpito la qualità della loro vita. Con "disgregazione ambientale" si intendono cambiamenti fisici, chimici e/o biologici nell'ecosistema (o nelle risorse di base) che lo rendono, temporaneamente o permanentemente, inadatto a supportare la vita umana (El-Hinnawi 1985, p. 4). Questa definizione base, fornita da Essam El-Hinnawi, andrebbe arricchita, per fornire un ventaglio più ampio delle possibili situazioni che coinvolgono i migranti ambientali. Come sostenuto da Graeme Hugo, non si possono mettere sullo stesso piano quanti fuggono da un evento disastroso improvviso, come un'eruzione vulcanica o uno tsunami, e quanti lasciano gradualmente le loro terre a causa dell'abbassamento del livello qualitativo del suolo. Per distinguere le due tipologie, Hugo si riferisce alla prima tipologia come "migrazioni involontarie" e alla seconda come "migrazioni volontarie". Tra le une e le altre, vi sarebbe una serie di tipologie di migrazioni differenti. Classificazione interessante è quella, per esempio dei cosiddetti anticipatory refugees, cioè di coloro che decidono di migrare prima che il livello di degradazione dell'area in cui vivono raggiunga i massimi livelli e li costringa, da un giorno all'altro, a vivere in maniera traumatica il trasferimento (Hugo 1996). Parlare di "migrazioni volontarie", in questo caso, non è necessariamente corretto, perché può trattarsi in ogni caso di una scelta obbligata. Lo testimonia il fatto che molti di questi migranti cercano di aiutare (attraverso le rimesse) coloro che sono rimasti nel paese d'origine, per garantire la sopravvivenza di comunità rurali anche in ambienti climaticamente instabili (David 1995, p. 18). Molti conservano la speranza di poter tornare a casa.

Alla mancanza di una definizione univoca utilizzata per indicare le migrazioni legate ai mutamenti climatici, si aggiunge anche la mancanza di un riconoscimento giuridico dei protagonisti di tali movimenti migratori. L'Organizzazione internazionale per le migrazioni e l'Intergovernmental Panel on Climate Change (IPCC) hanno sconsigliato I'uso del termine "rifugiato climatico" et similia, perché il rischio è quello di creare delle aspettative che, molto probabilmente, resterebbero deluse. Entrambi (con l'aggiunga del Refugee Policy Group) hanno preferito l'utilizzo dell'espressione "environmentally displaced

\footnotetext{
${ }^{6}$ Si vedano le possibili varianti, tra cui: "rifugiato ambientale", "eco migrante, migrante ambientale forzato", "rifugiato climatico", "sfollato ambientale", etc. (Castaldi 2014, p. 44).
} 
persons". Per garantire il riconoscimento giuridico della figura di rifugiato ambientale, sarebbe necessario un adeguamento della Convenzione di Ginevra ${ }^{7}$. Al momento, tale convenzione regola solo i casi relativi a conflitti bellici o persecuzioni. Ne consegue che coloro che scappano a causa di disastri ambientali sono costretti a vivere in condizioni di clandestinità nei paesi di destinazione. Soluzioni parziali sono state immaginate in alcuni paesi più virtuosi. La Nuova Zelanda rappresenta un buon esempio: meta preferita, insieme all'Australia, dei flussi migratori che partono dalla Polinesia (in cui intere isole rischiano di essere sommerse dal mare), ha introdotto dei permessi speciali per tutelare i nuovi arrivati. Tuttavia, è evidente che, di fronte a un problema che possiede tutte le caratteristiche per diventare strutturale, tali interventi sono destinati a risultare assolutamente insufficienti. Nell'Unione Europea, fatta eccezione per Finlandia e Svezia, sono fondamentalmente assenti politiche pubbliche ad hoc per affrontare la presenza di "migranti ambientali".

Un invito a sopperire a tale mancanza arriva dall'Ambasciatore William Lacy Swing, direttore generale dell'OIM:

"Now that climate change and migration are an integral part of the Paris agreement, we can justifiably speak of "climate migration" and "climate migrants". [...] We face major migration and refugee movements, and climate change is among the root causes of the record number of persons forced to migrate. By taking action to harness the positive potential of migration as an adaptation strategy to climate change we can support those who might need sooner or later to migrate with dignity. [...] Not all of these people will become climate migrants, but now is the time to get prepared in order to avoid future tragedies».

Questo invito da parte di Swing è certamente incoraggiante. Tuttavia, se è vero che vengono fatti passi in avanti sul piano dell'attenzione ai problemi di natura ecologica, è vero anche che spesso seguono immediati passi indietro: si pensi alla differente attitudine mostrata dalla precedente e dall'attuale presidenza statunitense rispetto al problema dei mutamenti climatici e alla disponibilità a rispettare l'Accordo di Parigi sul clima. Per quanto la questione ecologica sembri trovarsi quotidianamente al centro del dibattito, l'importanza che le si attribuisce quasi mai è pari a quella che si attribuisce all'economia. Mentre il mondo occidentale continua a cercare un modo per uscire dalla crisi che lo attanaglia dal 2008, tornando sempre sullo stesso modello di sviluppo - anziché metterlo in discussione -, la situazione delle aree svantaggiate continua a peggiorare.

\footnotetext{
${ }^{7}$ La Convenzione di Ginevra è nota anche come Convention Relating to the Status of Refugees o Refugee Convention.
} 


\section{Conclusioni}

Come si è visto anche nell'ultimo paragrafo, un conto è avere la percezione che esistano dei problemi di ordine ambientale, le cui conseguenze possono riversarsi sui singoli e su intere popolazioni; tutt'altra cosa sono il riconoscimento e la difesa dei loro diritti, minacciati dalle condizioni di rischio cui sono sottoposti a causa dei mutamenti climatici.

Posto che si riuscissero a migliorare significativamente e a rendere strutturali gli strumenti di tutela nei confronti dei migranti per motivazioni ecologiche, si tratterebbe in ogni caso di interventi "a valle". Ciò che resta necessario e urgente è lo spostamento dell'attenzione sulle cause reali di tali fenomeni (collocate "a monte") e sullo storico debito che una parte del mondo deve ancora saldare con l'altra parte. II modello di sviluppo (e di produzione) occidentale, che per decenni si è imposto come esempio da seguire per i paesi con caratteristiche culturali (ed economiche) completamente diverse, è tra i primi responsabili dè fenomeni di inquinamento e di cambiamento climatico, nonché della grave ingiustizia e diseguaglianza sociale tra i paesi del Nord e i paesi del Sud del mondo. II gap di potere tra i due raggruppamenti si configura anche in termini ecologici, laddove per potere intendiamo «la capacità di godere dei benefici ambientali e di far pagare ad altri i costì (Sachs - Santarius 2007, p. 76). La globalizzazione economica ha inciso fortemente su questo punto attraverso la separazione tra fasi "pulite" di produzione (che restano nei paesi industrialmente avanzati) e fasi "inquinanti" (che, è risaputo, vengono de-localizzate nei paesi più deboli anche dal punto di vista dei salari e delle tutele sociali). In altre parole, le società ricche esternalizzano i costi di produzione, anche in termini ambientali, scaricandoli su quelle povere.

Se, per lungo tempo, i paesi più ricchi hanno pensato di poter trarre solo vantaggi da questa organizzazione globale dell'economia, oggi hanno cominciato a percepire che il conto salato di scelte sbagliate verrà, in definitiva, presentato anche a loro. Solo un'inversione di termini - che riponga l'economia al suo posto, come mezzo della vita umana e non come fine ultimo -, potrà frenare la serie di mutamenti non solo immediati e improvvisi, ma anche graduali, che rischiano di rendere it pianeta terra non più abitabile dall'essere umano. Un tale capovolgimento implicherebbe: la fine del dominio delle relazioni economiche e produttive su tutte le altre, il ritorno all'enfatizzazione di elementi della vita umana diversi da quelli strettamente collegati alla dimensione materiale, una maggiore consapevolezza dell'importanza rivestita dalla tutela della nafura. Si tratterebbe di pervenire a una compenetrazione reciproca delle forme di organizzazione economica e sociale, tale da assicurare un'economia integrata fondata sulle idee di sviluppo umano e di giustizia sociale, quest'ultima declinata anche in termini ambientali (Dahrendorf 2003). 


\section{Riferimenti bibliografici}

- BENTHAM, Jeremy (1907 [1789]), Introduction to the Principles of Morals, Clarendon Press, Oxford.

BOLOGNA, Gianfranco (2005), Manuale della sostenibilità. Idee, concetti, nuove discipline capaci di futuro, Edizioni Ambiente, Milano.

CASTALDI, Flavia (2014), Le migrazioni ambientali: prime riflessioni geografiche, in Silvia Aru Andrea Corsale Marcello Tanca (a cura di), Percorsi migratori della contemporaneità. Forme, pratiche, territori, Cuec, Cagliari; pp. 4153.

CeSPI - Centro Studi di Politica Internazionale (a cura di), Cambiamenti climatici e governance della sicurezza: la rilevanza politica della nuova agenda internazionale, n. 16 maggio 2010. Milano.

COHEN, Daniel (2004), Tre lezioni sulla società postindustriale, Garzanti,

CONDORCET (de), Jean-Antoine Nicolas (1995 [1795]), I progressi dello spirito umano, Editori Riuniti, Roma.

DAHRENDORF, Ralf (2003), Quadrare il cerchio. Benessere economico, coesione sociale e libertà politica, Laterza, Bari.

DAVID, Rosalind (1995), Changing Places? Women, Resource Management and Migration in the Sahel. Case studies from Senegal, Burkina Faso, Mali and Sudan, SOS Sahel, London.

DIDEROT, Denis (1782 [1751-1776]), Droit naturel in D. Diderot - J.B. Le Rond d'Alembert (eds.), Encyclopédie ou Dictionnaire raisonné des sciences, des arts et des métiers, tome XI, Chez La sociétés Typographiques, Lausanne et Berne.

DOUGLAS LUMMIS, C. (1998), Uguaglianza in Wolfgang Sachs (a cura di), Dizionario dello sviluppo, Torino, Edizioni Gruppo Abele.

DOUGLAS, Mary (1995), Di fronte allo straniero. Una critica antropologica delle scienze sociali, in "ll Mulino", n. 1, pp. 5-24.

- DURANTE, Graziella (2009), La nuova carta dei poteri. Dispotismi, interessi e possibilità dell'eguaglianza, in Condorcet, Gli sguardi dell'illuminista. Politica e ragione nell'età dei lumi, a cura di Graziella Durante, Edizioni Dedalo, Bari.

EL-HINNAWI, Essam (1985), Environmental Refugees, Unep, Nairobi.

GALBRAITH, John Kenneth (1958), The Affluent Society, Houghton Mifflin Company, Boston-New York. Milano.

GALBRAITH, John Kenneth (1993), La cultura dell'appagamento, Rizzoli,

- $\quad$ HAQ, Mahbub ul (2003), Readings in Human Development, Oxford University Press, Oxford. 
- HIGH REPRESENTATIVE AND THE EUROPEAN COMMISSION TO THE EUROPEAN COUNCIL (2008), Climate Change And International Security, S1 13/08

HUGO, Graeme (1996), Environmental concerns and international migration in "International Migration Review", n. 30, 1996, pp. 105-131.

- $\quad$ KANT, Immanuel (1999 [1795]), Per la pace perpetua, Feltrinelli, Milano.

- KEYNES, John Maynard (1996), La fine del laissez faire e altri scritti, Bollati Boringhieri, Torino.

LATOUCHE, Serge (2005), Come sopravvivere allo sviluppo. Dalla decolonizzazione dell'immaginario economico alla costruzione di una società alternativa, Bollati Boringhieri, Torino.

- LATOUCHE, Serge (2000), La sfida di Minerva. Razionalità occidentale e ragione mediferranea, Bollati Boringhieri, Torino.

- NIADA, Marco (2006), Rapporto shock: economia mondiale minacciata dai cambiamenti climatici, ne «il Sole 24 ore», 30 ottobre 2006.

- SACHS Wolfgang (2000), Ambiente e giustizia sociale. I limiti della globalizzazione, Editori Riuniti, Roma.

- $\quad$ SACHS, Wolfgang - MOROSINI, Marco, (a cura di) (2011), Futuro sostenibile. Le risposte eco-sociali alle crisi in Europa, Edizioni Ambiente, Milano.

SACHS, Wolfgang - SANTARIUS, Tilman, (a cura di) (2007), Commercio e agricoltura. Dall'efficienza economica alla sostenibilità sociale e ambientale, EMI, Città di Castello (PG).

- $\quad$ SEN, Amartya (1988), Etica ed Economia, Laterza, Bari.

- $\quad$ SEN, Amartya (2000), Lo sviluppo è libertà, Mondadori, Milano.

- $\quad$ SEN, Amartya (2002), Globalizzazione e libertà, Mondadori, Milano.

SMITH, Adam (1973), Indagine sulla natura e le cause della ricchezza delle nazioni, ISEDI, Milano.

- $\quad$ UNEP (2009), From Conflict to Peacebuilding. The Role of Natural Resources and the Environment, Nairobi

- VOLTAIRE (de), François-Marie Arovet (1965 [1764]), Dizionario filosofico, a cura di M. Bonfantini, Giulio Einaudi Editore, Milano.

- ZENGHELIS, Dimitri (2017), La decarbonizzazione: innovazione ed economia dei cambiamenti climatici, in M. Mazzucato - M. Jacobs (a cura di), Ripensare il capitalismo, Laterza, Bari-Roma; pp. 275-306.

ZETTER, Roger (2015), Migration and environmental change: examining the relationship, in Science Communication Unit (ed.), Migration in response to environmental change, University of the West of England (UWE), Bristol. 